\title{
Advantages of $\mathrm{L}-3-\left[{ }^{18} \mathrm{~F}\right]$
} CrossMark fluoro-alpha-methyl tyrosine over 2- $\left[{ }^{18} \mathrm{~F}\right]$-fluoro-2-deoxyglucose in detecting liver metastasis during positron emission tomography scan

Sayaka Kodaira ${ }^{1 *}$, Takahito Nakajima², Yukiko Arisaka², Azusa Tokue ${ }^{2}$, Tetsuya Higuchi ${ }^{1}$ and Yoshito Tsushima ${ }^{1}$

\begin{abstract}
Purpose: We aimed to assess the usefulness of positron emission tomography (PET) using the amino acid tracer L-3-[18F] fluoro-alpha-methyl tyrosine (FAMT) in detecting metastatic liver lesions compared with 2-[18F]-fluoro2-deoxyglucose (FDG).
\end{abstract}

Methods: We included 24 patients with liver metastases who underwent both FDG-PET/computed tomography (CT) and FAMT-PET/CT. Maximum standardized uptake value (SUVmax) and tumor-to-liver parenchymal (T/L) ratio were analyzed to evaluate the correlation between FDG and FAMT uptakes in metastatic liver lesions; adenocarcinoma $(A C, n=21)$, squamous cell carcinoma (SCC, $n=23)$, neuroendocrine tumor (NET, $n=9$ ), and carcinoid tumor (CAR, $\mathrm{n}=6)$.

Results: We detected 59 lesions on performing either FDG-PET or FAMT-PET. NETs had significantly lower T/L ratios for FAMT (median, 1.00; range, 0.86-1.34) compared with those for FDG (median 2.86; range 1.70-6.13, p < 0.01). CAR tumors tended to reveal lower T/L ratios for FDG (median 1.10; range 0.78-1.92) than those for FAMT (median 1.80; range $0.80-2.34)$. Comparison of T/L ratios of SCC and AC revealed that FAMT in the metastatic liver lesions of SCC was higher than those of $A C(p<0.05)$.

Conclusion: FAMT-PET could detect metastatic liver lesions from various cancers, except NET.

Keywords: Liver metastasis, L-3-[ $\left[{ }^{18} \mathrm{~F}\right]$ fluoro-alpha-methyl tyrosine, 2-[ $\left.{ }^{18} \mathrm{~F}\right]$-fluoro-2-deoxyglucose, Positron emission tomography

\section{Background}

Involvement of the liver as a site of cancer metastasis causes significant problems in morbidity and mortality. Because liver dysfunction due to the poor control of liver metastasis would shorten patient survival period, the assessment of metabolic function of the liver with

\footnotetext{
*Correspondence: sayaka.kodaira@gmail.com

${ }^{1}$ Department of Diagnostic Radiology and Nuclear Medicine, Gunma University Graduate School of Medicine, 3-39-22, Showa, Maebashi, Gunma 371-8511, Japan

Full list of author information is available at the end of the article
}

metastasis is an important prognostic tool (Wiering et al. 2005; Bonanni et al. 2014; Fernandez et al. 2004).

Positron emission tomography (PET) using an ${ }^{18} \mathrm{~F}$-labeled glucose analog, 2-[ $\left.{ }^{18} \mathrm{~F}\right]$-fluoro-2-deoxyglucose (FDG) is the most sensitive imaging method to detect malignant lesions that utilize glucose at greater rates than normal tissue because of an increase in both glucose transport and metabolism through the glucose transporter (GLUT1) (Juweid and Cheson 2006). Because metastatic liver lesions have little or no glucose-6-phosphatase, FDG is trapped within the cell (Mamede et al. 2005; Okazumi et al. 1992). Therefore, FDG-PET scans 
reveal that metastatic liver lesions have a high FDG uptake.

Many clinical studies revealed the feasibility of FDGPET for diagnosing, staging, treatment monitoring, and detecting recurrent malignant tumors (Fischer et al. 2009; Pieterman et al. 2000; Kostakoglu and Goldsmith 2003). The detection of liver metastasis from colorectal and other gastrointestinal cancers has been evaluated in many reports; most of them addressed the very high sensitivity of FDG-PET in detecting liver metastasis compared with other modalities, such as computed tomography $(\mathrm{CT})$ and magnetic resonance imaging (MRI), by the administration of conventional contrast agents (Kinkel et al. 2002; Bipat et al. 2005). Currently, MRI using gadolinium-ethoxybenzyl-diethylenetriamine pentaacetic acid (Gd-EOB-DTPA) has shown very high detectability for hepatic lesions. However, FDG-PET still has the advantage for detecting liver lesions. In contrast, the sensitivity of FDG-PET was not good, particularly for small liver lesions. The reasons would be the poor spatial resolution of PET and that FDG uptake in normal parenchymal tissue would obscure small liver lesions without high-FDG uptake. In addition, differentiation between inflammation and tumor lesions is difficult for FDG-PET (Nishii et al. 2013; Yamada et al. 1998; Morita et al. 2013). Nevertheless, FDG-PET still plays an important role in detecting metastatic liver lesions in a clinical setting.

It also is well known that amino acid tracers also accumulate in cancer lesions. In contrast to FDG, amino acid tracers do not accumulate in inflammatory lesions and the specificity of amino acid tracers to malignant lesions is reliable compared with that of FDG(Inoue et al. 1999, 2001). Tumor cells require amino acid transporters such as L-amino acid transporter type 1 (LAT1) (Kaira et al. 2008; Namikawa et al. 2015). In our facility, we developed L-3- $\left[{ }^{18} \mathrm{~F}\right]$ fluoro-alpha-methyl tyrosine (FAMT) as an amino acid PET tracer(Tomiyoshi et al. 1997). The specific accumulation of FAMT in malignant tumors has been evaluated in a clinical setting and has been demonstrated to be useful in the diagnosis of various types of malignant tumors. With regard to the detection of lesions in the liver, because the most investigated amino acid tracers, ${ }^{11} \mathrm{C}$-methionine (MET) and anti-1-amino-3-18Ffluorocyclobutane-1-carboxylic acid (FACBC), accumulate in both cancerous and normal liver cells, their uptake in the normal liver tissue is strong enough to obscure the accumulation in metastatic lesions. In contrast, FAMT uptake in a normal liver has been known to be low, making FAMT a good candidate to detect metastatic liver lesions.

In this study, the accumulations of FDG and FAMT in metastatic liver lesions were compared and correlated with their respective histological features.

\section{Methods}

\section{Patients}

This retrospective study included 24 patients (17 men and seven women; aged $32-85$ years; mean 67 years) with advanced cancer complicated by liver metastases who had undergone both FDG-PET/CT and FAMT-PET/CT between August 2007 and November 2014. The inclusion criteria were as follows: $(1)<1$-month interval between FDG-PET/CT and FAMT-PET/CT scans and (2) none of the metastatic lesions received treatment. Patients with duodenal pleomorphic sarcoma $(\mathrm{n}=1)$, malignant lymphoma $(\mathrm{n}=2)$, and lung small cell carcinoma $(n=3)$ were excluded from this study because the number of patients with liver metastasis for each group was only one. Blood samples were taken before tracer injection and an acceptable blood sugar level $(<200 \mathrm{mg} / \mathrm{dl})$ was confirmed. This study was reviewed and approved by our institutional review board, and informed consent was obtained from all patients.

\section{PET tracer administration}

Both FDG and FAMT were synthesized in the cyclotron facility of our institute; FAMT was synthesized according to the method of Tomiyoshi et al. Briefly, L-alpha-methyltyrosine was fluorinated by $\left[{ }^{18} \mathrm{~F}\right]$-acetylhypofluoride, and the separation and purification of FAMT were performed by a remote control system (Tomiyoshi et al. 1997). Patients were intravenously injected with FAMT $(5 \mathrm{MBq} / \mathrm{kg})$ and FDG $(5 \mathrm{MBq} / \mathrm{kg})$ after fasting for $>6 \mathrm{~h}$.

\section{Image acquisition}

PET/CT images were acquired at $1 \mathrm{~h}(60 \pm 5 \mathrm{~min})$ after injection using a Discovery STE PET/CT scanner (GE Healthcare, Milwaukee, WI) or a Biograph $16 \mathrm{PET} /$ CT scanner (Siemens, Malvern, PA) with 700-mm field of view (FOV) and a slice thickness of $3.27 \mathrm{~mm}$. Threedimensional (3D) data acquisition was performed for 3 min per bed position, followed by image reconstruction with the $3 \mathrm{D}$-ordered-subsets expectation maximization (3D-OSEM) method. Segmented attenuation was corrected by X-ray CT (140 kV, 120-240 mAs) to produce $128 \times 128$ matrix images. CT images were reconstructed using a conventional filtered back projection method. Axial full width at half-maximum (FWHM) at $1 \mathrm{~cm}$ from the center of FOV was $5.6 \mathrm{~mm}$; the z-axis FWHM at $1 \mathrm{~cm}$ from the center of FOV was $6.3 \mathrm{~mm}$. Intrinsic system sensitivity was $8.5 \mathrm{cps} / \mathrm{kBq}$ for $3 \mathrm{D}$ acquisition. Both the PET scanners were regularly calibrated with a phantom and their standardized uptake value (SUV) accuracy was routinely evaluated to ensure that the values produced were comparable. Patients were scanned from the thigh to the head in the arms-down position. No intravenous contrast material was administered for CT scanning. 
Limited breath holding at normal expiration was used during $\mathrm{CT}$ to avoid motion-induced artifacts and allow co-registration of CT and PET images in the area of the diaphragm.

\section{Data analysis}

PET/CT images acquired using FDG and FAMT were interpreted by a single experienced nuclear medicine physician and were analyzed using a syngo MI Workplace (VA60C, Siemens AG, Munich, Germany). The resolution of the reconstructed images was approximately $5 \mathrm{~mm}$ at FWHM.

To evaluate the distribution of both the PET tracers, the regions of interest (ROIs) were manually placed on each tumor lesion in the liver with assistance of CT images on the same slices, as well as on the normal liver parenchyma, back muscle, and mediastinum, which represented a blood pool by two experienced nuclear medicine physicians. SUVs in ROIs were calculated using the following formula: SUV $=$ [radioactive concentration in $\mathrm{ROI}(\mathrm{MBq} / \mathrm{g})] /[$ injected dose $(\mathrm{MBq}) /$ patient's body weight $(\mathrm{g})$ ].

Side-by-side review and analysis of radioactive images were performed to confirm that SUV was derived from the same lesion on both the PET scans. In this study, we employed SUVmax, which was defined as the peak SUV on the pixel with the highest count within ROI.

All tumor lesions were categorized by the histological examination of the primary lesion or the liver biopsy specimen as adenocarcinoma (AC); squamous cell carcinoma (SCC); neuroendocrine tumor (NET; i.e., pheochromocytoma, malignant endocrine tumor, and paraganglioma); and carcinoid tumor (CAR). To evaluate the correlation between FDG and FAMT uptakes in liver lesions, three SUVmax-related parameters were analyzed; these were (1) SUVmax itself, (2) tumor-to-liver parenchymal (T/L) ratio, and (3) tumor-to-blood pool $(\mathrm{T} / \mathrm{B})$ ratio.

\section{Statistical analysis}

The correlation between FDG and FAMT uptakes in the metastatic liver lesions in each histological group was evaluated by linear regression analysis. The Mann-Whitney $U$ test was performed to evaluate the differences between each SUVmax parameter of FAMT and FDG. For all statistical analyses, $\mathrm{p}$ value $<0.05$ was considered statistically significant.

\section{Results}

The primary tumors were esophageal carcinoma $(\mathrm{n}=10)$, lung carcinoma $(\mathrm{n}=7)$, pancreatic carcinoma $(n=2)$, rectal carcinoid $(n=2)$, and one patient each for primary lesions in the gingiva, adrenal gland, and carotid body (Table 1). The median interval between FDG-PET and FAMT-PET was 7.6 days (range $1-30$ days).

\section{Tracer uptake in metastatic liver lesions and liver parenchymal tissue}

A total of 59 lesions were detected on either FDG-PET or FAMT-PET in this study. Two lesions were not detected in FDG-PET/CT but were detected in FAMT-PET/CT. Fourteen lesions demonstrated opposing results with those of PET/CT; for these lesions, ROIs were carefully drawn while referring to PET with another tracer and enhanced CT images. On the basis of histological examinations, primary lesions were divided into four forms (Table 1): SCC $(\mathrm{n}=23), \operatorname{AC}(\mathrm{n}=21)$, NET $(\mathrm{n}=9)$, and $\operatorname{CAR}(\mathrm{n}=6)$.

SUVmax in these lesions ranged from 2.40 to 19.01 (median 6.16) for FDG and from 1.02 to 3.85 (median 1.85) for FAMT. As shown in Fig. 1, uptake of FAMT in metastatic lesions was significantly lower than that of FDG ( $\mathrm{p}<0.001$ ), whereas liver parenchymal tissue demonstrated an almost double SUVmax of FDG compared with that of FAMT $(\mathrm{p}<0.001)$; the median SUVmax of ${ }^{18} \mathrm{~F}$-FDG and ${ }^{18} \mathrm{~F}$-FAMT in liver parenchymal tissue was 2.61 (range 1.36-4.50) and 1.39 (0.88-2.32), respectively.

\section{Correlation between FDG and FAMT by each parameter}

Scatter plots between FDG and FAMT by each parameter are shown in Fig. 2. No significant correlations in SUVmax $(r=0.167, \mathrm{p}=0.207) ; \mathrm{T} / \mathrm{L}$ ratio $(\mathrm{r}=0.208$, $\mathrm{p}=0.114)$; and $\mathrm{T} / \mathrm{B}$ ratio $(\mathrm{r}=0.043, \mathrm{p}=0.744)$ were observed. The distributions indicated that (1) CAR lesions had low FDG uptake and relatively high FAMT uptake; (2) NET showed low FAMT uptake and relatively high FDG uptake; (3) the T/L ratios of SCC tended to be higher than those of AC. Only two lesions of AC showed high FDG uptakes compared with SCC; these two lesions were derived from esophageal cancer.

\section{Distribution of $\mathrm{T} / \mathrm{L}$ ratio in each histological category}

The distribution of $\mathrm{T} / \mathrm{L}$ ratio demonstrated some tendencies depending on the histological category, particularly for NET and CAR (Fig. 3). NETs had significantly lower $\mathrm{T} / \mathrm{L}$ ratios for FAMT uptake (median 1.00; range 0.861.34) compared with those for FDG uptake (median 2.86; range 1.70-6.13) ( $\mathrm{p}<0.01$, Fig. 4a). CAR tumors tended to reveal lower T/L ratios for FDG uptake (median 1.10; range $0.78-1.92$ ) than those for FAMT uptake (median 1.80; range $0.80-2.34$ ), but this was not significant $(\mathrm{p}=0.345$, Fig. 4b). 
Table 1 Characteristics of patients with liver metastasis

\begin{tabular}{|c|c|c|c|c|c|c|}
\hline No. & Age (years) & Sex & Primary tumor & $\begin{array}{l}\text { Lesion } \\
\text { number }\end{array}$ & Size $(\mathrm{cm})$ & Histological type \\
\hline 1 & 59 & M & Pancreas & 3 & $1.8-7.2$ & Adenocarcinoma \\
\hline 2 & 64 & $\mathrm{~F}$ & Lung & 3 & $2.4-3.1$ & Adenocarcinoma \\
\hline 3 & 83 & M & Rectum & 3 & $2.0-11.0$ & Carcinoid \\
\hline 4 & 67 & $\mathrm{~F}$ & Rectum & 3 & $8.8-12.4$ & Carcinoid \\
\hline 5 & 61 & M & Esophagus & 2 & $2.1-3.1$ & Squamous cell carcinoma \\
\hline 6 & 73 & M & Esophagus & 3 & $1.8-7.7$ & Squamous cell carcinoma \\
\hline 7 & 60 & $\mathrm{~F}$ & Lung & 1 & 5.3 & Adenocarcinoma \\
\hline 8 & 70 & M & Esophagus & 3 & $1.0-1.7$ & Squamous cell carcinoma \\
\hline 9 & 73 & M & Lung & 3 & $1.9-2.0$ & Adenocarcinoma \\
\hline 10 & 66 & M & Lung & 3 & $2.0-3.0$ & Squamous cell carcinoma \\
\hline 11 & 53 & M & Lung & 1 & 3.1 & Adenocarcinoma \\
\hline 12 & 77 & M & Esophagus & 3 & $1.6-2.2$ & Endocrine cell carcinoma \\
\hline 13 & 57 & M & Esophagus & 3 & $7.5-11.8$ & Adenocarcinoma \\
\hline 14 & 65 & $\mathrm{~F}$ & Adrenal gland & 3 & $1.1-2.6$ & Malignant pheochromocytoma \\
\hline 15 & 67 & M & Esophagus & 1 & 2.3 & Squamous cell carcinoma \\
\hline 16 & 67 & $\mathrm{~F}$ & Esophagus & 3 & $8.3-10.6$ & Squamous cell carcinoma \\
\hline 17 & 74 & M & Lung & 3 & $1.6-2.0$ & Adenocarcinoma \\
\hline 18 & 85 & M & Gingiva & 3 & $2.4-4.8$ & Squamous cell carcinoma \\
\hline 19 & 32 & M & Lung & 1 & 1.6 & Adenocarcinoma \\
\hline 20 & 53 & $\mathrm{~F}$ & Carotid body & 3 & $2.4-4.4$ & Paraganglioma \\
\hline 21 & 84 & M & Esophagus & 1 & 2.8 & Squamous cell carcinoma \\
\hline 22 & 81 & $\mathrm{~F}$ & Esophagus & 3 & $2.6-6.3$ & Squamous cell carcinoma \\
\hline 23 & 56 & M & Pancreas & 3 & $4.3-5.2$ & Adenocarcinoma \\
\hline 24 & 78 & M & Esophagus & 1 & 10.0 & Squamous cell carcinoma \\
\hline
\end{tabular}

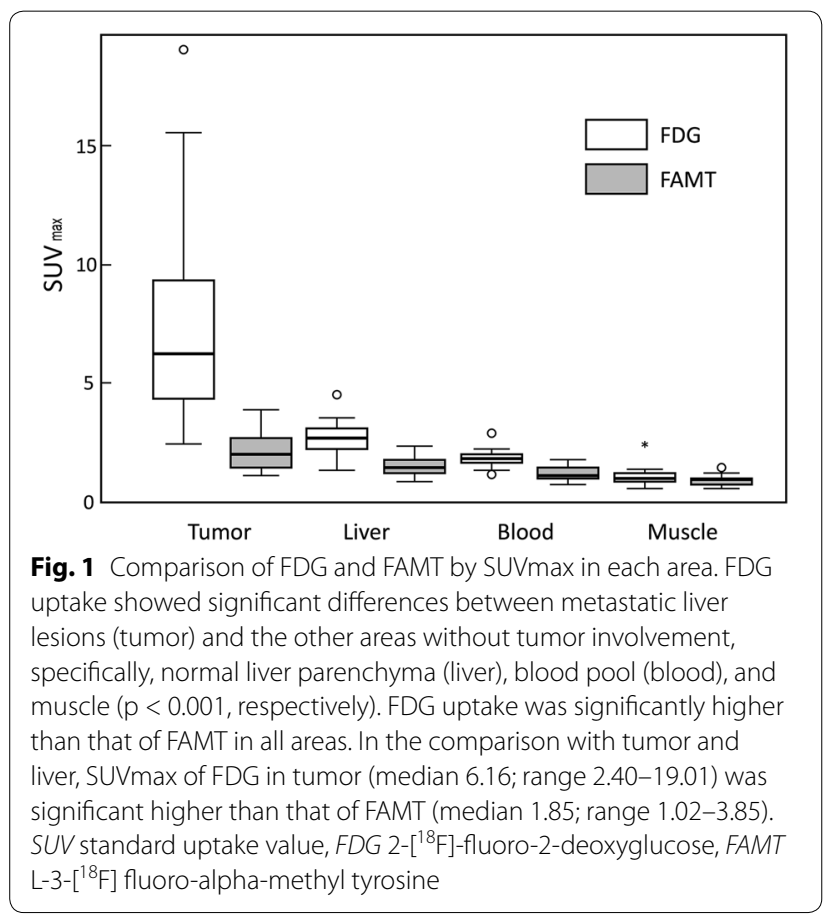

\section{Comparison of FDG and FAMT uptakes between SCC} and AC (Figs. 5, 6)

The T/L ratio of SCC had median values of 3.50 (range 1.09-6.02) on FDG-PET and 1.76 (range 0.92-2.69) on FAMT-PET. The T/L ratio of AC had median values of 2.28 (range 1.43-6.96) on FDG-PET and 1.26 (range 0.80-1.81) on FAMT-PET. Comparison of T/L ratios of SCC and AC revealed that FAMT uptake in the metastatic liver lesions of SCC was higher than that of AC $(\mathrm{p}<0.01)$. In contrast, FDG uptakes of SCC and AC were not significantly different $(\mathrm{p}=0.235)$.

\section{Discussion}

The respective uptake of FAMT was lower in both metastatic liver lesions and normal tissues compared with that of FDG. However, most liver metastases were recognized on FAMT-PET images, except NET lesions. Differential diagnosis among various tissues using $\mathrm{T} / \mathrm{L}$ ratio might be useful, particularly for comparison between SCC and AC on FAMT-PET. In FDG and FAMT combinations, tissues tended to be distributed on the basis of their histology (Fig. 2). 

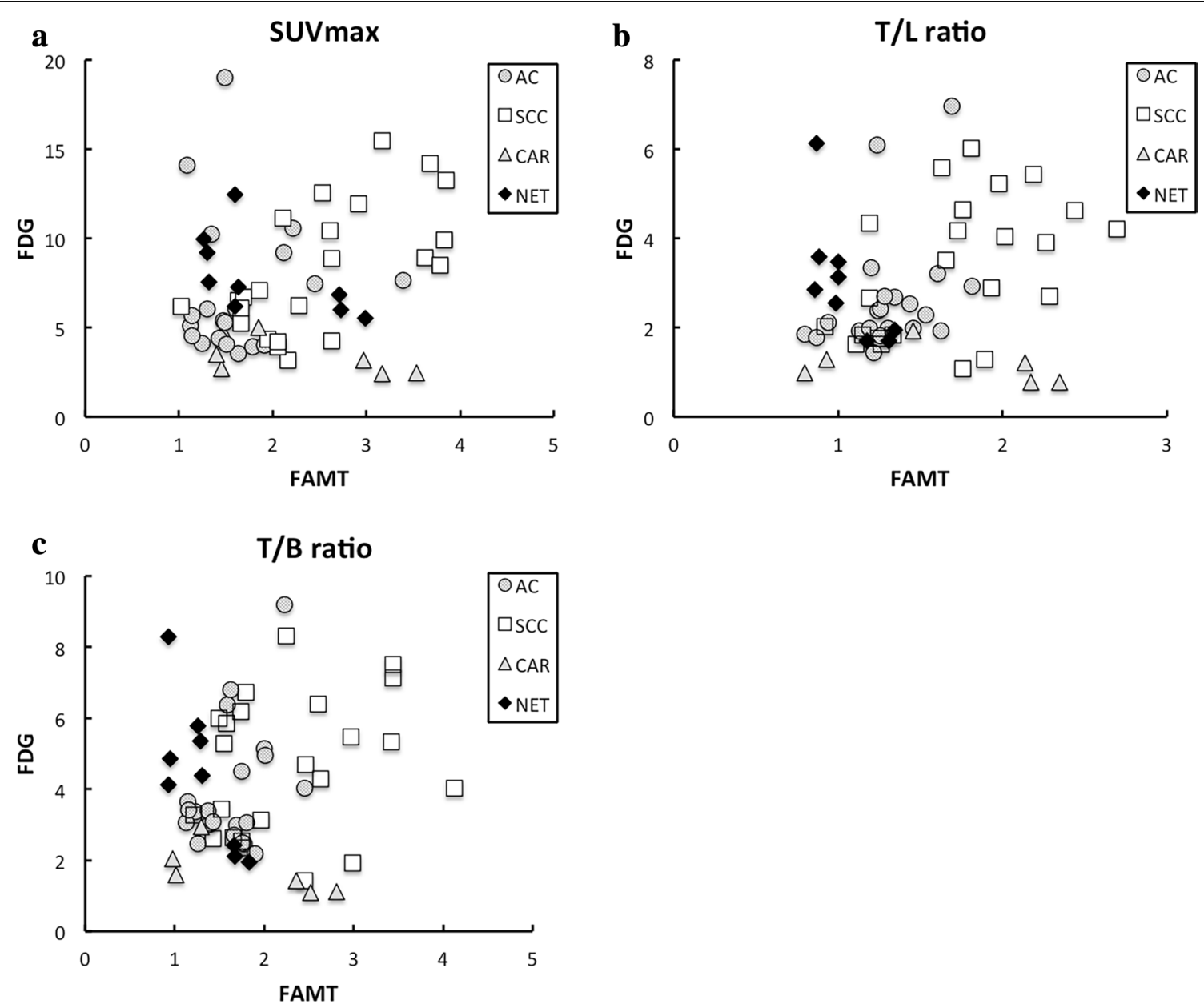

Fig. 2 Dot plots of SUVmax, T/L ratio, T/B ratio, and T/M ratio. Xaxis shows the SUVmax of FAMT and the $Y$ axis shows the SUVmax of FDG. No significant correlations of total lesions in a SUVmax, b T/L ratio, and $\mathbf{c} T / B$ ratio were observed $(r=0.167, p=0.207 ; r=0.208, p=0.114 ; r=0.043$, $p=0.744$, respectively). T/L ratio differed according to histological feature. SUV standard uptake value, FDG 2-[18F]-fluoro-2-deoxyglucose, FAMT L-3$\left[{ }^{18} \mathrm{~F}\right]$ fluoro-alpha-methyl tyrosine, T/L tumor-to-liver parenchymal, T/B tumor-to-blood pool, SCC squamous cell carcinoma, AC adenocarcinoma, NET neuroendocrine tumor and pheochromocytoma, CAR carcinoid tumor

Both FDG and FAMT uptakes for SCC were relatively higher than those for AC. Only two lesions of AC in one patient showed higher SUVmax compared with that in SCC; however, excluding these two lesions, the T/L ratio of FDG revealed no significant difference between SCC and AC ( $\mathrm{p}=0.063)$. This patient was the only case of esophageal adenocarcinoma. We speculated that this cancer lesion might have included adenosquamous components. Further investigations of LAT1 expression based on histology will be required to demonstrate the utility of FDG-PET or FAMT-PET for differentiating between SCC and AC. FDG uptakes of CAR tumors had relatively low $\mathrm{T} / \mathrm{L}$ ratios, resulting in difficulty to differentiate metastatic liver lesions from liver parenchymal tissues on FDG-PET. In contrast, CAR tumors were detected on FAMT-PET and FAMT-PET was superior to FDG-PET in this point.
In this study, FDG uptake in the liver parenchymal tissues ranged from 1.36 to 4.50 , and the median value of SUVmax (2.60) was already over the upper limit of the general threshold between benign and malignant lesions. FDG uptake in the liver parenchyma was higher than that of FAMT because glucose was metabolized and stored in the liver. In general, hepatocytes have glucose-6-phosphatase that can reverse FDG-6-phosphate to FDG, thus reducing FDG uptake in the hepatocyte (Torizuka et al. 1995; Khan et al. 2000; Yen et al. 2004). Therefore, FDG accumulation was relatively lower than what was expected of a glucose analog.

The differences between FDG and FAMT may be because of not only metabolism but also retention. Because FDG was taken up by GLUT1 and trapped into cells after phosphorylation, it accumulated in a linear 

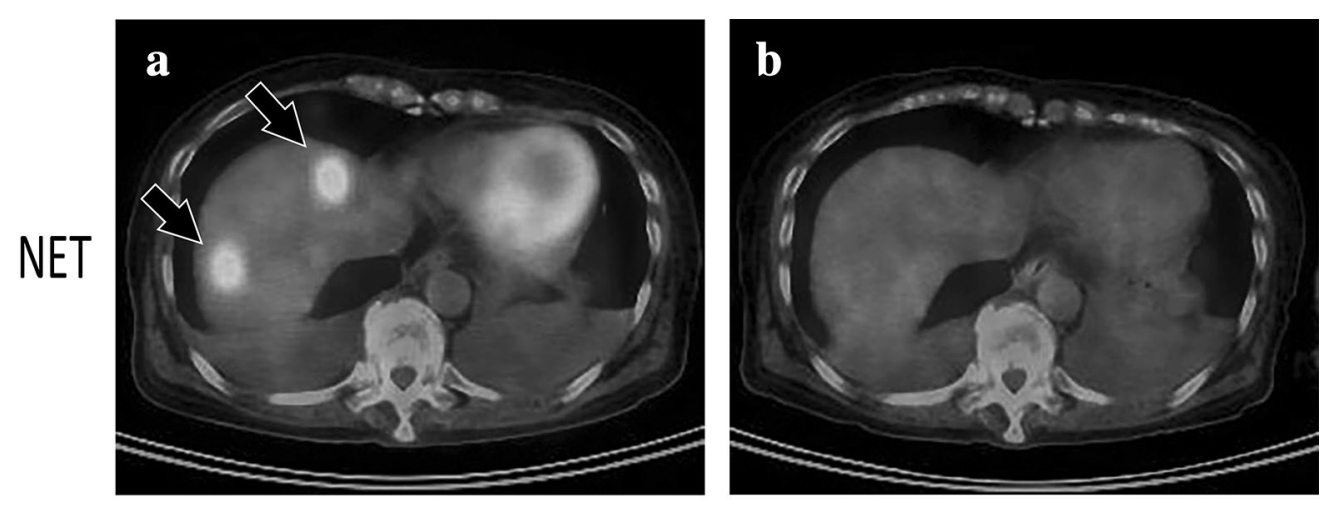

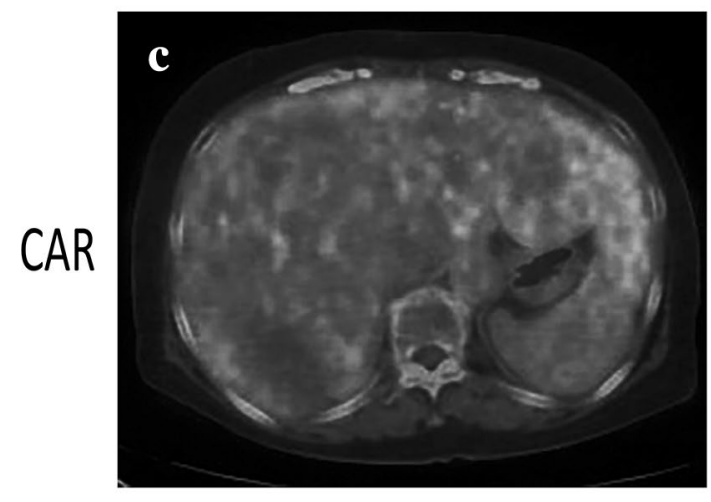

FDG

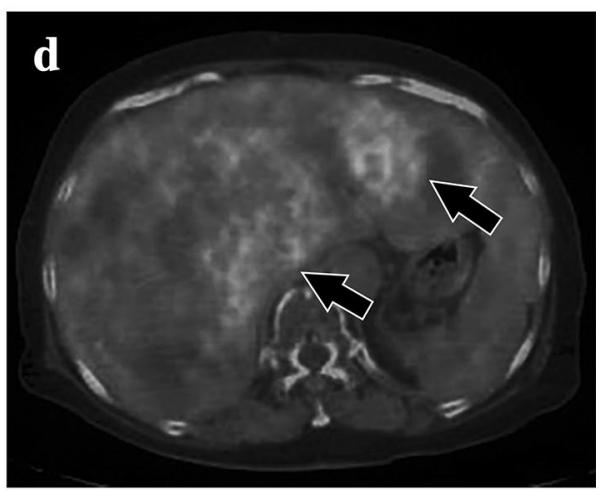

FAMT

Fig. 3 PET/CT fusion images with either FDG or FAMT administration for the same patients with NET or CAR. For a patient with NET, FDG PET images showed high spotty accumulations (arrow) in the liver (a). No FAMT uptakes were seen in the liver (b). For a patient with CAR, FDG PET showed diffuse uptake for normal liver parenchyma without uptakes for tumor lesion in the liver (c). FAMT PET showed some faint massive uptakes (arrow) of FAMT in the liver (d). FDG 2-[18 F]-fluoro-2-deoxyglucose, FAMT L-3- $\left[{ }^{18} \mathrm{~F}\right]$ fluoro-alpha-methyl tyrosine, T/L tumor-to-liver parenchymal, CAR carcinoid tumor, NET neuroendocrine tumor and pheochromocytoma

fashion and was eventually retained. Conversely, after FAMT transport into cells by LAT1, relatively rapid clearance of FAMT can be observed in even tumor cells; this means that LAT1 function of FAMT transport was not one way but was reversible. These mechanisms may explain the lower SUVmax of FAMT.

Alpha-methylation of FAMT reduces liver accumulation and increases renal excretion, whereas the analog of FAMT, L-tyrosine, has been well known to be one of the aromatic amino acids that are metabolized in the liver. This is an advantage of FAMT over the most commonly studied MET, which strongly accumulates in both the normal and metastatic liver tissues (Wiriyasermkul et al. 2012). Another analog of tyrosine and a well-known amino acid tracer, $\mathrm{O}-\left(2-{ }^{18} \mathrm{~F}\right.$-fluoroethyl)L-tyrosine (FET), has been shown to have low accumulation in murine liver and a higher specificity than FDG (Ishiwata et al. 2004). Although FET would be a good candidate for detecting liver metastasis, most reports on its application were for brain tumors, and there has been no report on liver metastasis.
Wiriyasermkul et al. (2012) revealed that FAMT had two potential benefits: one was the reduced uptake in the liver and another was the specific transport via LAT1 without the need to go through other types of amino acid transporters, such as the system ASC amino acid transporter-2 (ASCT2). The details of the mechanisms for uptake of FAMT or methionine in normal liver are not clear; however, FAMT is transported only via $\mathrm{L}$-amino acid transporter-1 (LAT1), while methionine is transported via LAT-1, LAT-2, and some other transporters (Singhal et al. 2008). LAT1 specificity of FAMT may increase the possibility of detecting metastatic liver lesions.

Nevertheless, because FDG strongly accumulated in the metastatic liver lesions, $\mathrm{T} / \mathrm{L}$ ratios were still higher than those of FAMT. In this study, T/L ratio indicated the specific features based on histological origins. Although FAMT uptake was detected in metastatic liver lesions from NETs, FDG accumulation was very high (median; 7.28, ranging from 5.50 to 12.45). Our clinical experiences revealed that FAMT-PET had a difficulty in the 

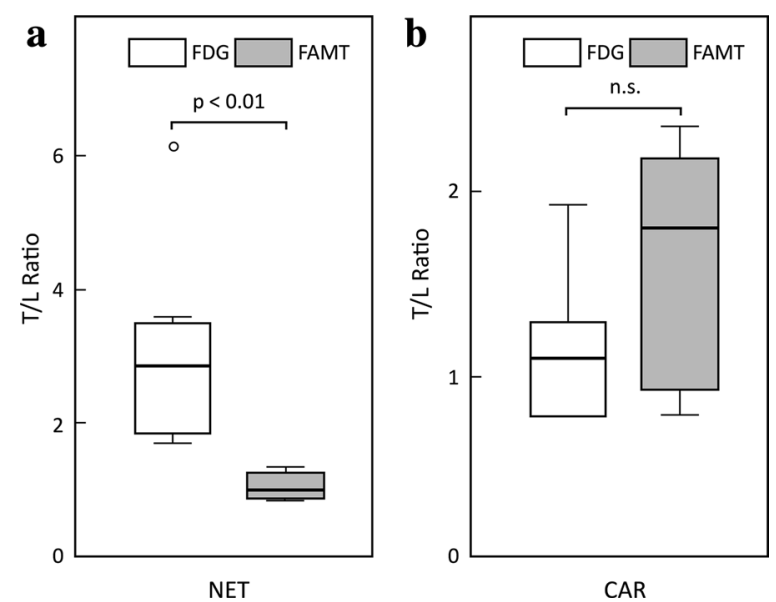

Fig. 4 Comparison between FDG an FAMT in terms of T/L ratio in NET lesions and CAR lesions. a T/L ratio of FAMT uptake was significantly lower than that of FDG in the group of NET lesions $(p<0.01)$. b $T / L$ ratio of FAMT (median 1.80) was slightly higher than that of FDG (median 1.10), but not significant ( $p=0.345$ ). FDG 2-[ $\left.{ }^{18} \mathrm{~F}\right]$-fluoro-2-deoxyglucose, FAMT L-3-[ $\left.{ }^{18} \mathrm{~F}\right]$ fluoro-al pha-methyl tyrosine, $T / L$ tumor-toliver parenchymal, CAR carcinoid tumor, NET neuroendocrine tumor and pheochromocytoma
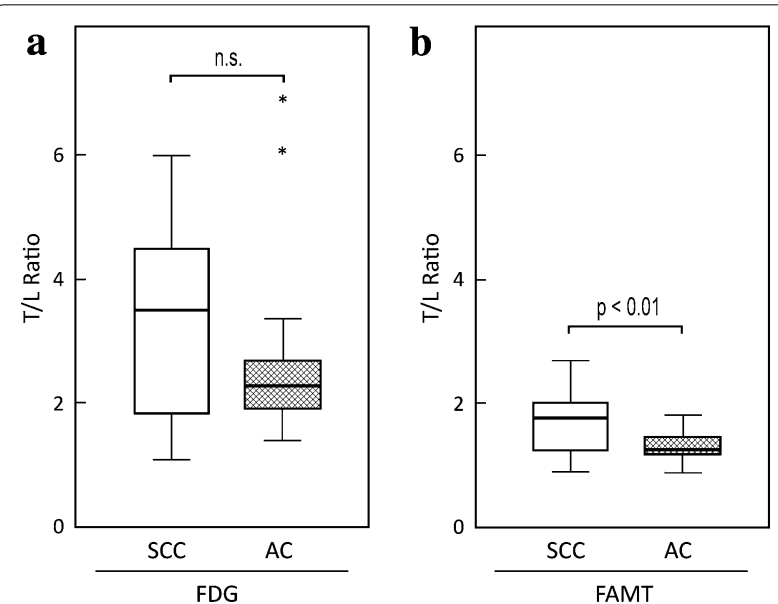

Fig. 5 Comparison of T/L ratios in FDG and FAMT between SCC and AC. a FDG uptakes in liver metastasis from SCC were higher than those from AC $(p<0.05)$. b FAMT uptakes for SCC and AC were not significantly different $(p=0.235)$. FDG 2-[18 F]-fluoro-2-deoxyglucose, FAMT L-3- $\left[{ }^{18} \mathrm{~F}\right]$ fluoro-alpha-methyl tyrosine, $T / L$ tumor-to-liver parenchyma, T/B tumor-to-blood pool, SCC squamous cell carcinoma, AC adenocarcinoma

detecting pheochromocytoma lesions (unpublished data), whereas FDG had an advantage. In contrast, the detection of liver metastasis from CAR tumors using FDG-PET was difficult because FDG uptake was low; thus, for these lesions, FAMT-PET could be more useful. According to the International Union Against Cancer

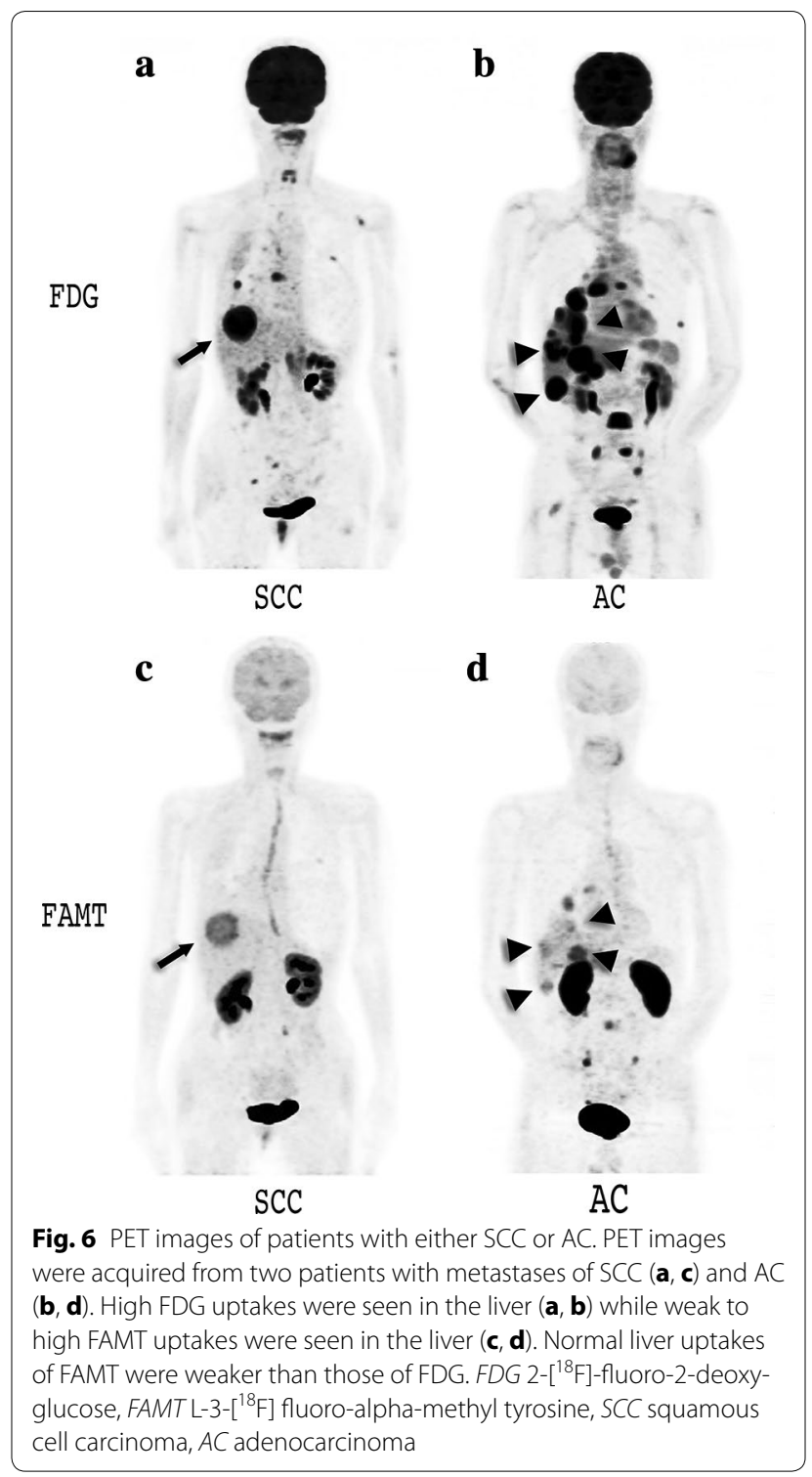

classification of neuroendocrine tumors, CAR belongs to G1 and NET belongs to G2 or G3. Because the uptake of FDG generally reflects the grading of tumor differentiation, FDG-PET should have shown higher accumulations in NET than in CAR. Low-grade tumors, such as NET, show a high uptake of FDG compared with well-differentiated tumors such as CAR. In our case, two lesions were detected using only FAMT-PET not FDG-PET.

The limitation of this study was that patients with colorectal cancer were not included. Although most metastatic liver lesions from colorectal cancer would be AC, our AC group included metastasis from lung cancers. However, some reports revealed high expressions of GLUT1 and high FDG uptake in SCC (Yen et al. 2004; 
Fletcher et al. 2008). Our comparison between SCC and $\mathrm{AC}$ may not be different from that for colorectal cancer. FAMT-PET was performed not for conventional clinical examinations but for study purposes; this study did not include metastatic liver lesions from colorectal cancers. Therefore, the number of patients who underwent both FDG-PET and FAMT-PET was small, and this would be a limitation for statistical analysis. In further investigations, the time of PET acquisition after tracer administration can be changed to an earlier time. It would have an advantage with obtaining high FAMT uptakes in liver lesions. However, the high T/L ratio would be required to depict liver lesions more clearly. Because of the background signals, including normal liver uptakes is another important factor and should be investigated for an optimal time.

\section{Conclusion}

There is no general advantage in use of FAMT-PET over that offered by FDG for detecting liver metastasis. However, since FAMT-PET could detect metastatic liver lesions from many types of cancers, except NET, histological tendencies were shown by comparisons between PET studies of FDG and FAMT.

\section{Authors' contributions}

SK, YA, and TN conceived and designed the experiments and performed data acquisition,analysis, interpretation, and statistical analysis. SK, TN wrote the manuscript. All of the authors contributed to revise the manuscript. All authors read and approved the final manuscript.

\footnotetext{
Author details

${ }^{1}$ Department of Diagnostic Radiology and Nuclear Medicine, Gunma University Graduate School of Medicine, 3-39-22, Showa, Maebashi, Gunma 371-8511, Japan. ${ }^{2}$ Department of Molecular Imaging, Gunma University Graduate School of Medicine, 3-39-22, Showa, Maebashi, Gunma 371-8511, Japan.
}

\section{Acknowledgements}

This research was supported by the nuclear medicine department of Gunma University Hospital.

\section{Competing interests}

The authors declare that they have no competing interests.

\section{Ethical statement}

Informed consent was obtained from all individual participants included in the study.

Received: 2 March 2016 Accepted: 22 April 2016

Published online: 13 May 2016

\section{References}

Bipat S, van Leeuwen MS, Comans EFI, Pijl MEJ, Bossuyt PMM, Zwinderman AH et al (2005) Colorectal liver metastases: CT, MR imaging, and PET for diagnosis-meta-analysis. Radiology 237:123-131

Bonanni L, De'liguori Carino N, Deshpande R, Ammori BJ, Sherlock DJ, Valle JW et al (2014) A comparison of diagnostic imaging modalities for colorectal liver metastases. Eur J Surg Oncol 40:545-550
Fernandez FG, Drebin JA, Linehan DC, Dehdashti F, Siegel BA, Strasberg SM (2004) Five-year survival after resection of hepatic metastases from colorectal cancer in patients screened by positron emission tomography with F-18 fluorodeoxyglucose (FDG-PET). Ann Surg 240:438-447

Fischer B, Lassen U, Mortensen J, Larsen S, Loft A, Bertelsen A et al (2009) Preoperative staging of lung cancer with combined PET-CT. N Engl J Med 361:32-39

Fletcher JW, Djulbegovic B, Soares HP, Siegel BA, Lowe VJ, Lyman GH et al (2008) Recommendations on the use of 18F-FDG PET in oncology. J Nucl Med 49:480-508

Inoue T, Shibasaki T, Oriuchi N, Aoyagi K, Tomiyoshi K, Amano S et al (1999) $18 \mathrm{~F}$ alpha-methyl tyrosine PET studies in patients with brain tumors. J Nucl Med 40:399-405

Inoue T, Koyama K, Oriuchi N, Alyafei S, Yuan Z, Suzuki H et al (2001) Detection of malignant tumors: whole-body PET with fluorine 18 alpha-methyl tyrosine versus FDG — preliminary study. Radiology 220:54-62

Ishiwata K, Kawamura K, Wang W, Furumoto S, Kubota K, Pascali C et al (2004) Evaluation of O- $\left[{ }^{11} \mathrm{C}\right]$ methyl-L-tyrosine and $\mathrm{O}-\left[{ }^{18} \mathrm{~F}\right]$ fluoromethyl-L-tyrosine as tumor imaging tracers by PET. Nucl Med Biol 31:191-198

Juweid ME, Cheson BD (2006) Positron-emission tomography and assessment of cancer therapy. N Engl J Med 354:496-507

Kaira K, Oriuchi N, Imai H, Shimizu K, Yanagitani N, Sunaga N et al (2008) L-type amino acid transporter 1 and CD98 expression in primary and metastatic sites of human neoplasms. Cancer Sci 99:2380-2386

Khan M, Combs C, Brunt E (2000) Positron emission tomography scanning in the evaluation of hepatocellular carcinoma. J Hepatol 32:792-797

Kinkel K, Lu Y, Both M, Warren RS, Thoeni RF (2002) Detection of hepatic metastases from cancers of the gastrointestinal tract by using noninvasive imaging methods (US, CT, MR imaging, PET): a meta-analysis. Radiology 224:748-756

Kostakoglu L, Goldsmith SJ (2003) 18F-FDG PET evaluation of the response to therapy for lymphoma and for breast, lung, and colorectal carcinoma. J Nucl Med 44:224-239

Mamede M, Higashi T, Kitaichi M, Ishizu K, Ishimori T, Nakamoto Y et al (2005) $\left[{ }^{18} \mathrm{~F}\right] \mathrm{FDG}$ uptake and PCNA, Glut-1, and Hexokinase-II expressions in cancers and inflammatory lesions of the lung. Neoplasia. 7:369-379

Morita M, Higuchi T, Achmad A, Tokue A, Arisaka Y, Tsushima Y (2013) Complementary roles of tumour specific PET tracer ${ }^{18} \mathrm{~F}$-FAMT to ${ }^{18} \mathrm{~F}$-FDG PET/ CT for the assessment of bone metastasis. Eur J Nucl Med Mol Imaging 40:1672-1681

Namikawa M, Kakizaki S, Kaira K, Tojima H, Yamazaki Y, Horiguchi N et al (2015) Expression of amino acid transporters (LAT1, ASCT2 and XCT) as clinical significance in hepatocellular carcinoma. Hepatol Res 45:1014-1022

Nishii R, Higashi T, Kagawa S, Kishibe Y, Takahashi M, Yamauchi H et al (2013) Diagnostic usefulness of an amino acid tracer, a-[N-methyl- 11C]methylaminoisobutyric acid (11C-MeAIB), in the PET diagnosis of chest malignancies. Ann Nucl Med 27:808-821

Okazumi S, Isono K, Enomoto K, Kikuchi T, Ozaki M, Yamamoto H et al (1992) Evaluation of liver tumors using fluorine-18-fluorodeoxyglucose PET: characterization of tumor and assessment of effect of treatment. J Nucl Med 33:333-339

Pieterman R, van Putten J, Meuzelaar J, Mooyaart E, Vaalburg W (2000) Preoperative staging of non-small-cell lung cancer with positron-emission tomography. N Engl J Med 343:254-261

Singhal T, Narayanan TK, Jain V, Mukherjee J, Mantil J (2008) 11C-L-methionine positron emission tomography in the clinical management of cerebral gliomas. Mol Imaging Biol 10:1-18

Tomiyoshi K, Amed K, Muhammad S, Higuchi T, Inoue T, Endo K et al (1997) Synthesis of isomers of $18 \mathrm{~F}$-labelled amino acid radiopharmaceutical: position 2- and 3-L-18F-alpha-methyltyrosine using a separation and purification system. Nucl Med Commun 18:169-175

Torizuka T, Tamaki N, Inokuma T, Magata Y, Sasayama S, Yonekura Y et al (1995) In vivo assessment of glucose metabolism in hepatocellular carcinoma with FDG-PET. J Nucl Med 36:1811-1817

Wiering B, Krabbe PFM, Jager GJ, Oyen WJG, Ruers TJM (2005) The impact of fluor-18-deoxyglucose-positron emission tomography in the management of colorectal liver metastases. Cancer 104:2658-2670

Wiriyasermkul P, Nagamori S, Tominaga H, Oriuchi N, Kaira K, Nakao H et al (2012) Transport of 3-fluoro-L-a-methyl-tyrosine by tumor-upregulated 
L-type amino acid transporter 1: a cause of the tumor uptake in PET. J Nucl Med 53:1253-1261

Yamada Y, Uchida Y, Tatsumi K, Yamaguchi T, Kimura H, Kitahara H (1998)

Fluorine-18-fluorodeoxyglucose and carbon-11 methionine evaluation of

lymphadenopathy in sarcoidosis. J Nucl Med 39:1160-1166
Yen T-C, See L-C, Lai C-H, Yah-Huei CW, Ng K-K, Ma S-Y et al (2004) 18F-FDG uptake in squamous cell carcinoma of the cervix is correlated with glucose transporter 1 expression. J Nucl Med 45:22-29

\section{Submit your manuscript to a SpringerOpen ${ }^{\circ}$ journal and benefit from:}

- Convenient online submission

\section{- Rigorous peer review}

- Immediate publication on acceptance

- Open access: articles freely available online

- High visibility within the field

- Retaining the copyright to your article 